\title{
The New Man and his films: a glance at how contemporary Romanian cinema traces the recent past
}

\author{
Marina Vargau \\ Université de Montréal, Montréal, H3T 1N8, Canada \\ marinavargau@yahoo.ca
}

\begin{abstract}
Romanian filmmakers who have won prizes at international film festivals in the first decade of twenty-first century belong to the same generation: that of the New Man, a paradigm brought forth by Ceausescu's communist regime. My analysis will, in the first place, discuss the documentary Nascuti la comanda. Decreteii (Children of the Decree, 2004) by Florin Iepan. In this film we will follow the traces of the two traumatisms that marked this generation: the ban of abortion through decree by the State in 1966 - a moment which announces the birth of the New Man - and the anti-communist Romanian revolution of December 1989, in which most of the participants and victims belonged to the generation born under the order of the Party. The new generation of Romanian filmmakers have shown through their films that this New Man is actually rethinking the past and its traumas. The question of abortion and of its consequences will be discussed in relation to the film 4 luni, 3 saptamani si 2 zile (4 months, 3 weeks and 2 days, 2007) by Cristian Mungiu and to the short Visul lui Liviu (Liviu's Dream, 2004) by Corneliu Porumboiu, while a polyphonic image of the Romanian revolution will be put into question in three films: Cum am celebrat sfarsitul lumii (How I celebrated the end of the world, 2006) by Catalin Mitulescu, Hartia va fi albastra (The paper will be blu, 2006) by Radu Muntean and A fost sau n-a fost? (12:08 East of Bucarest, 2006) by Corneliu Porumboiu.
\end{abstract}

\section{Introduction}

Le documentaire Le sang des bêtes, réalisé en 1949 par Georges Franju, jette un regard cinématographique objectif sur le monde sanglant des abattoirs situés aux portes de Paris. Les images,

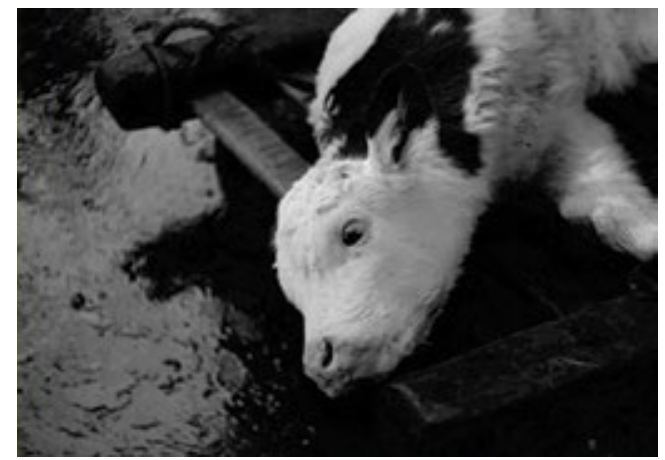

Figure 1. Le sang des bêtes, 1949, Georges Franju

d'une violence excessive, montrent en direct la tuerie des animaux. On voit l'agonie, le sang qui éclate, les derniers spasmes avant la mort, puis l'écorchement et le tranchement des corps. Siegfried Kracauer [1] relie ces images de violence contre les animaux aux images des corps humains torturés, montrées dans les films réalisés dans les champs de concentration nazis. Selon lui, grâce à ces images, on redonne à l'horreur, caché sous les voiles de la panique et de l'imagination, sa visibilité.

En 1985, en Roumanie, Copel Moscu réalise un film documentaire de 12 minutes qui présente une ferme avicole. Le documentaire, intitulé $V a$ veni o zi («Le jour viendra»), est interdit (il sera présenté pour la première fois en 1992, après la chute du régime communiste) parce que Moscu a le courage de suggérer à travers des images brutales sur le processus de sélection des poussins ce qui se cache derrière un autre processus de sélection, celui de l'«Homme nouveau»: l'extermination des enfants avec des déficiences durant le régime communiste de Ceausescu. Pour reprendre le commentaire de 
Kracauer, grâce à ce documentaire, Moscu donne la visibilité à une horrifiante réalité roumaine.

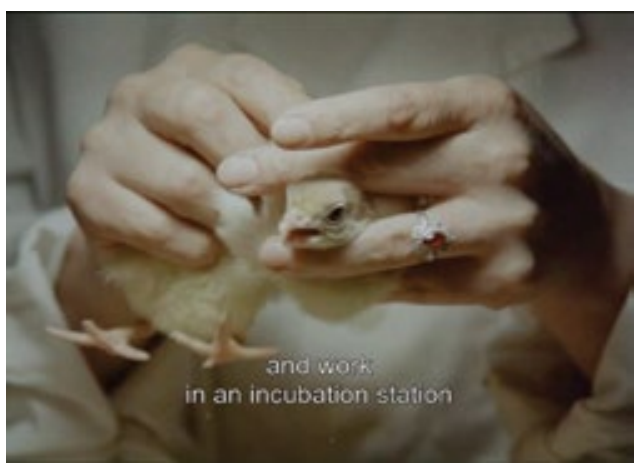

Figure 2. Va veni o zi, 1985, Copel Moscu

En 2005, Florin Iepan inclue quelques séquences de ce film dans son documentaire Nascuti la comanda. Decreteii («Nés à la commande. Les enfants $d u$ décret»), pour introduire des images absolument terrifiantes et choquantes, montrant les enfants trouvés en 1990, trois mois après la Révolution, dans l'orphelinat de Cighid (Roumanie), qui fonctionne comme centre d'extermination. Ces

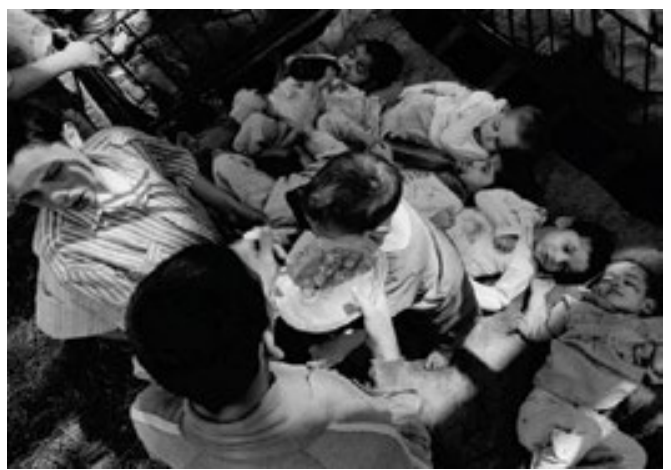

Figure3. Nascuti la comanda. Decreteii, 2003, Florin Iepan

enfants, pour la plupart des «conséquences» du décret 770 par lequel l'avortement est interdit, après avoir été «sélectionnés» par des commissions de médecins et trouvés inaptes pour la vie, sont abandonnés à l'orphelinat de Cighid, où, sans bénéficier de soins médicaux, d'aliments, de chauffage, d'électricité, d'un espace adéquat, sont laissés tout simplement mourir. Parfois, dans les pièces lugubres, ces enfants maigres, malades, sousdéveloppés, sont mangés vifs par les rats.

Les enfants de Cighid représentent le visage caché, monstrueux, d'une expérimentation sociale Étatique qui a duré 23 ans et qui avait comme but la création d'une nouvelle génération, endoctrinée politiquement et performante: la génération de l'«Homme nouveau».

\section{Le décrèt 770 de 1966 et ses conséquences}

Pour mieux comprendre cette réalité de la Roumanie communiste, il faut remonter au fil du temps, jusqu'en 1966, l'an qui marque l'interdiction de l'avortement et, implicitement, l'acte de naissance de l'«Homme nouveau». Le but de l'interdiction de l'avortement par le décret 770 de 1966 est l'augmentation de la population du pays pour des raisons économiques. L'État demande à chaque femme fertile de donner naissance à quatre ou cinq enfants, la reproduction est entièrement politisée et la maternité devient la responsabilité fondamentale des femmes roumaines envers l'État. Sans moyens de contraception, les femmes deviennent une proie pour l'État, qui les réduit à la fonction d'outils de reproduction. Un an après le décret, pris au dépourvu, sans contraceptifs et avec une vie sexuelle normale, les Roumains réussissent la «performance» d'augmenter deux fois le taux de natalité dans un seul an. Quand, en 1969, le Roumain no. 20000000 est né, Ceausescu est fier de son projet.

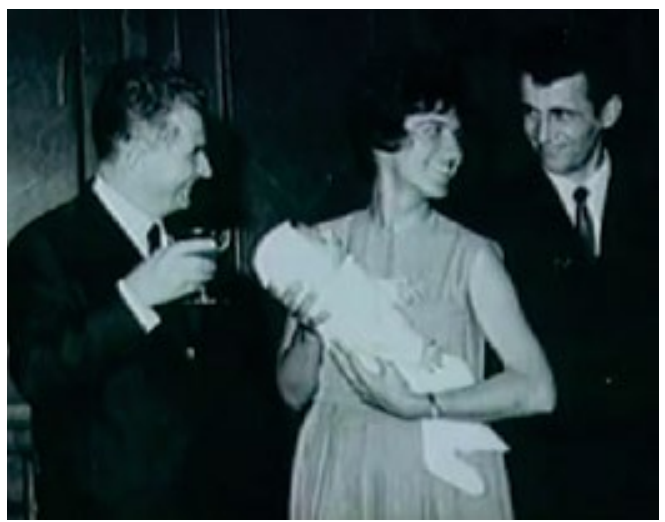

Figure 4: Ceausescu et les parents de l'enfant choisi pour être le 20000000 Roumain.

Les seules modalités de contrecarrer les doléances du Pouvoir sont l'abstinence comme moyen de contraception et l'avortement clandestin, 
fait dans des conditions impropres et dangereuses. Ceux qui, d'une façon ou d'une autre, sont impliqués dans l'avortement illégal sont poursuivis dans la justice et subissent diverses peines, parmi lesquelles la prison est la plus exemplaire.

Quand en 1973, pour la première fois depuis l'adoption du décret, la natalité commence à baisser, Ceausescu prend de nouvelles mesures coercitives. Les représentants de la Milice et de la Procurature occupent des positions dans les hôpitaux, même dans les salles d'opération, et ont le droit de vie et de mort sur les femmes qui se sont faites des avortements. Ces femmes, interrogées, battues, en absence de collaboration, sont laissées à mourir, les médecins n'ayant pas la permission d'intervenir. En 1984, le régime prend d'autres mesures. Pour surveiller encore mieux la vie intime des femmes, Ceausescu impose les contrôles gynécologiques périodiques mensuels, subis obligatoirement par les femmes à leurs places de travail, dans le but de découvrir la grossesse le plus tôt possible et de suivre son déroulement. Par cette mesure, les femmes n'ont aucune possibilité de s'échapper à leur obligation primordiale devant l'État : devenir mères. Mal nourris, dans le froid et le noir des appartements communistes, sans contraceptifs et aucune éducation sexuelle, les Roumains sont obligés de se reproduire pour augmenter la population du pays.

La chute du régime nationaliste et communiste de Ceausescu met fin à ce cauchemar, car le décret 770 est abrogé le 25 décembre 1989. À l'heure de son triste bilan, le projet de Ceausescu de créer l'«Homme Nouveau» est considéré aujourd'hui l'une des plus grandes expériences sociales de l'histoire de l'humanité : dans 23 ans, plus de 2000 000 d'enfants non-désirés ont été nés et plus de 10 000 femmes sont mortes suite aux avortements clandestins. Paradoxalement, les «enfants du décret», nés à la commande et qui, théoriquement devraient servir les intérêts du régime politique de Ceausescu, ont été les premiers à sortir dans les rues en décembre 1989 et, toujours eux, ont constitué la plupart des victimes durant la Révolution anticommuniste et anti-Ceausescu.

\section{L’Homme nouveau au cinéma}

Les deux traumatismes qui ont marqué la naissance, le devenir et la mort symbolique de la génération de l'«Homme nouveau», notamment l'interdiction de l'avortement en 1966 et la
Révolution roumaine de 1989, sont questionnées par la plupart des cinéastes qui font partie de ce qu'on appelle «la nouvelle vague roumaine» et qui sont les récipiendaires de nombreux prix dans les festivals internationaux à partir de 2005. Biologiquement, ils font partie de la génération de 1'«Homme nouveau», (Mungiu et Iepan sont nés en 1968, Muntean en 1971, Mitulescu en 1972, Porumboiu en 1975), génération au-dessus de laquelle plane une question angoissante: est-ce que ces enfants nés durant cette période ont été désirés par leurs familles ou ils sont des decretei, des conséquences du décret 770 ?

Cette génération des cinéastes roumains montre dans ses films que l'«Homme nouveau» d'autrefois repense aujourd'hui le passé récent et ses traumas, notamment l'avortement clandestin et ses conséquences, comme dans le film 4 luni, 3 saptamani si 2 zile (4 mois, 3 semaines et 2 jours) de Cristian Mungiu, gagnant du Prix Palme d'Or en 2007, et le court Visul lui Liviu (Le rêve de Liviu, 2004) de Corneliu Porumboiu, tandis que la révolution roumaine est questionnée dans trois films sortis en 2006 : Cum mi-am petrecut sfarsitul lumii (Comment j'ai fêté la fin du monde) de Catalin Mitulescu, Hartia va fi albastra (Le papier sera bleu) de Radu Muntean et $A$ fost sau $n$-a fost ? (12:08 A l'est de Bucarest) de Corneliu Porumboiu. Ces films se constituent en autant de questions générationnelles et une recherche de repères.

Le film 4 mois, 3 semaines et 2 jours de Cristian Mungiu raconte une journée de la vie de deux étudiantes à la Polytechnique. L'une d'entre elle, Găbita (Laura Vasiliu), enceinte, doit subir un avortement illégal le même jour. L'autre, Otilia (Anamaria Marinca), sa collègue de faculté et de chambre dans les résidences universitaires, fait tout pour l'aider. L'avortement illégal se passe dans une chambre d'hôtel et les jeunes filles doivent payer

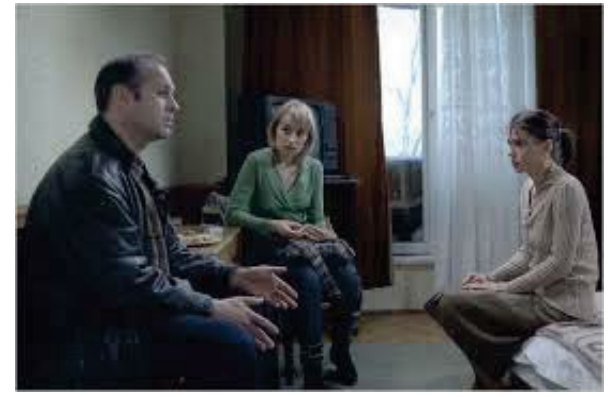

Figure 5. 4 luni, 3 saptamani si 2 zile, 2007, Cristian Mungiu 
d'avance le service de Monsieur Bebe (Vlad Ivanov), le faiseur d'anges, en acceptant d'être violées l'une après l'autre.

Lui-même enfant du décret, comme le déclare ouvertement, Cristian Mungiu a voulu raconter d'une manière neutre une histoire réelle passée dans les dernières années de dictature. Critiqué par certains pour avoir montré trop le fœtus avorté, le cinéaste se défend. Le traumatisme subi par sa génération des decretei, ces enfants non-désirés par leurs parents, est vif et douloureux et il doit être montré, devenir visible.

Un court-métrage réalisé par Corneliu Porumboiu, Visul lui Liviu, précède comme thématique le film de Mungiu, en montrant les conséquences de la loi contre l'avortement vues par ceux qui, d'une manière paradoxale, doivent être reconnaissants à Ceausescu pour leur existence. Liviu (Dragos Bucur), lui-même enfant du décret, fait un cauchemar récurrent qu'il est incapable de se rappeler. À la fin du film, il se souvient : chaque

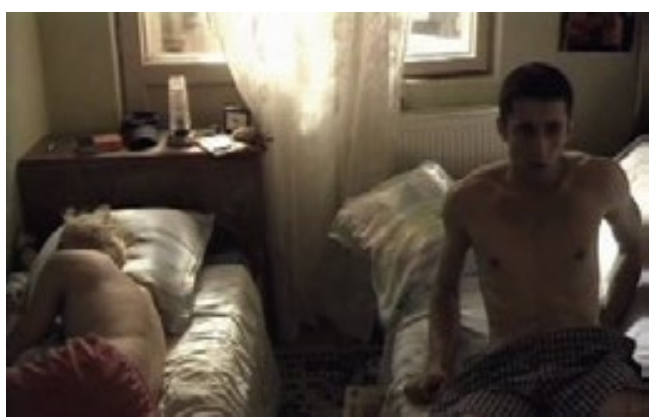

Figure 6. Visul lui Liviu, 2004, Corneliu Porumboiu

matin, il rêve de son frère jamais né qui, une chandelle dans la main, erre comme un fantôme dans le noir des souterrains, mais qui, finalement, conduira le protagoniste vers la lumière.

La Révolution roumaine est questionnée dans trois films sortis en 2006. Le film Comment j'ai passé la fin du monde de Catalin Mitulescu présente la vie d'une famille ordinaire qui vit dans une banlieue de Bucarest durant les deux dernières années de dictature de Ceausescu. La vie d'Eva (Dorotheea Petre) est bouleversée par un incident. L'écrasement accidentel d'un buste en plâtre de Ceausescu et le refus de l'adolescente de donner des explications entrainent son exclusion de l'organisation officielle des jeunes communistes (en roumain, UTC) et du lycée. Sa vie change complètement et, à partir de ce moment-là, elle a un seul désir: de quitter son pays. Après des entraînements physiques pour pouvoir franchir le Danube en nageant, la fille renonce au dernier moment à son plan et retourne chez soi, à sa famille et son frère, l'émouvant personnage Lalalilu (Timotei Duma), et aussi au garçon qu'elle aime. $\mathrm{La}$

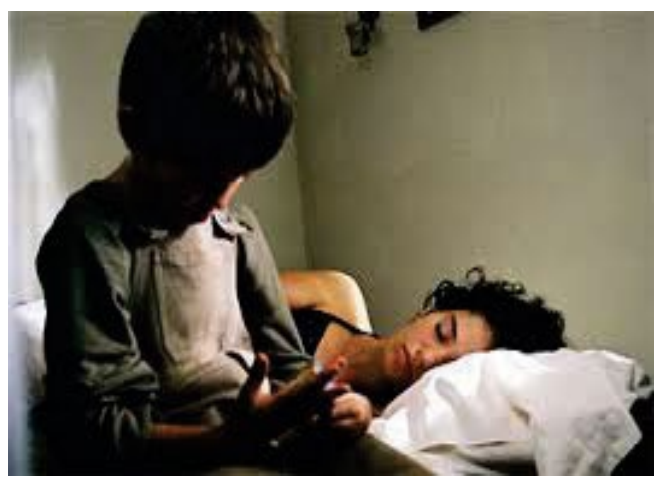

Figure 7. Cum mi-am petrecut sfarsitul lumii, 2006, Catalin Mitulescu

fin du film montre alternativement des imagesdocument - transmissions directes de la télévision roumaine durant la Révolution de 1989 - et des séquences fictives montrant comment la petite communauté décrite dans le film a vécu ce moment historique. Enfant unique d'un père méprisé par la communauté à cause de son travail comme délateur pour la police secrète roumaine, le jeune Alexandru (Ionut Becheru) veut montrer à la fille aimée et à tous son courage et qu'il peut se battre pour la liberté. Mais il se fait tué durant le chaos qui règne dans les rues de Bucarest et devient l'une de nombreuses victimes innocentes de la Révolution.

Le film Le papier sera bleu, signé par Radu Muntean, est entièrement consacré à cet événement et surprend très bien la confusion générale de ces jours-là de décembre quand, dans les rues de Bucarest, il y avait des feux d'armes partout, quand on ne savait pas si l'armée roumaine et la milice étaient du côté des révolutionnaires ou de l'État communiste, qui donnait des ordres, qui tirait contre qui, qui étaient les terroristes. Le protagoniste collectif du film est une patrouille de milice qui, la nuit de 22 décembre 1989, erre dans un char de combat par un Bucarest chaotique, avec une 
mission ambiguë : défendre, surveiller, intervenir ou bien se cacher et fuir. Idéaliste, le jeune soldat qui fait son service militaire obligatoire déserte son poste et fuit pour défendre la Télévision, la place la plus

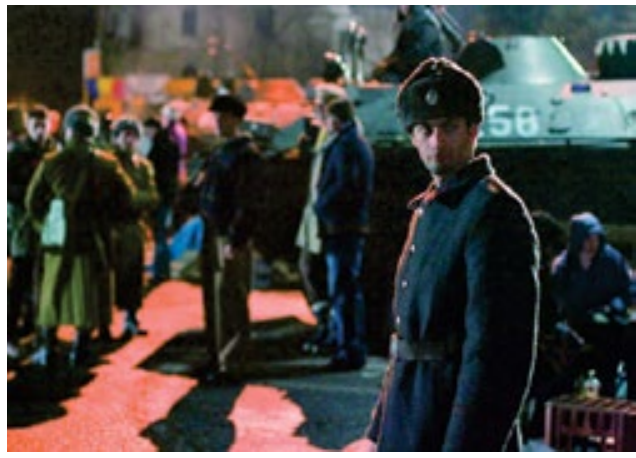

Figure 8 : Hartia va fi albastra, 2006, Radu Muntean

dangereuse à ce moment-là, mais sans y arriver à cause du chaos dans les rues. On voit des soldats qui tirent contre d'autres soldats, on voit comment le jeune homme et le tzigane rencontré par hasard sont pris pour des terroristes, et, finalement, quand le jour se lève et on a l'impression que le cauchemar nocturne a pris fin, on voit une scène absurde : la patrouille, faute d'avoir le bon mot de passe, est exterminée par d'autres jeunes soldats en service militaire obligatoire. Ce tragique imbroglio est dû au mal fonctionnement des transmissions d'émission-réception à cause des brouillages et des interférences. Pour que cet événement devienne plus absurde et incompréhensible, les images de la tuerie, les dernières dans la chronologie des évènements racontés, sont montrées au début du film, sans aucune introduction. Les spectateurs sont consternés: on a du mal à comprendre le pourquoi et comment on est arrivé dans une telle situation. Mais la réalité de ces jours et nuits-là de décembre 1989 est encore plus inexplicable et plus problématique, même regardée à une distance de plus de vingt ans.

Dans un registre parodique et absurde, le film 12h8. A l'est de Bucarest de Corneliu Porumboiu joue sur la même confusion, restée irrésolue après des années. «Y a-t-il eu, oui ou non, une révolution dans notre ville?», se demande le réalisateur d'un débat télévisée seize ans après décembre 1989. Dans cette émission, transmise en direct sur le poste local d'une petite ville moldave, «à l'est de Bucarest», Jderescu (Teo Corban), ex-ingénieur textile devenu propriétaire du poste local de télévision, présentateur et politologue, Manescu (Ioan Sapdaru), professeur d'histoire et ivrogne notoire dans la ville, et Piscoci (Mircea Andreescu), un vieux monsieur à la retraite qui toute sa vie a fait le Père Dugel (le substitut laïque du Papa Noel durant le communisme) essaient d'élucider l'énigme des événements de décembre 1989 dans leur ville.

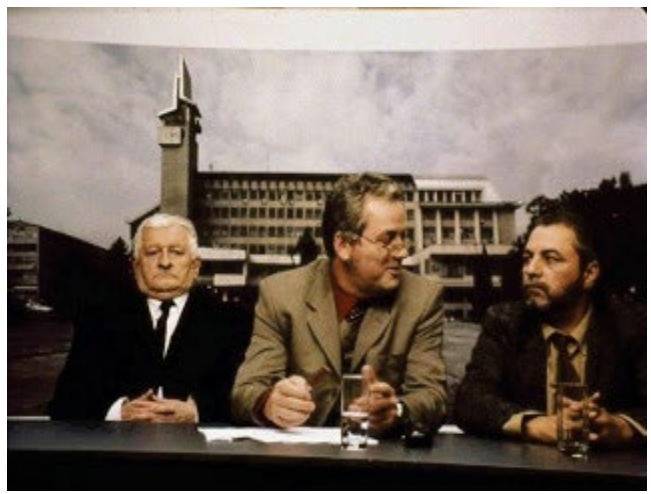

Figure 9. A fost sau n-a fost ?, 2006, Corneliu Porumboiu

Sous cette question réitérée avec insistance dans le film se cache le questionnement concernant les événements réels de décembre 1989 en Roumanie. Dans un article publié en 2007, Ruxandra Cesereanu [2] essaie de faire le point sur ces événements, en énumérant les trois interprétations «classiques» - «une révolution pure»; «un complot manipulé de l'extérieur»; «un coup d'État interne»-, et en avançant une autre hypothèse, selon laquelle «il s'est agi d'un événement hybride entre révolution spontané, révolution de palais et/ou complot de l'extérieur»".

$\mathrm{Si}$, aujourd'hui encore, le mystère et l'ambiguïté planent sur ces événements, il est certain que, depuis 1990, les centres de grandes villes de la Roumanie, parmi lesquels Bucarest, Timisoara, Brasov, sont devenus des lieux de mémoire, réaménagés pour abriter des cimetières où sont enterrés les héros de la Révolution, pour la plupart des jeunes qui sont descendus dans la rue en décembre 1989 et qui ont été tué dans le chaos et la confusion générales. Si les vrais coupables ne sont encore nulle part, les morts sont là, enterrés. Dans le documentaire Né à la commande, à la demande : 
«Qui est-il l'«Homme nouveau»?» la réponse est une autre question: «Est-il un homme avec des qualités extraordinaires ou seulement une personne ordinaire qui a le courage de payer avec sa vie pour la liberté?»

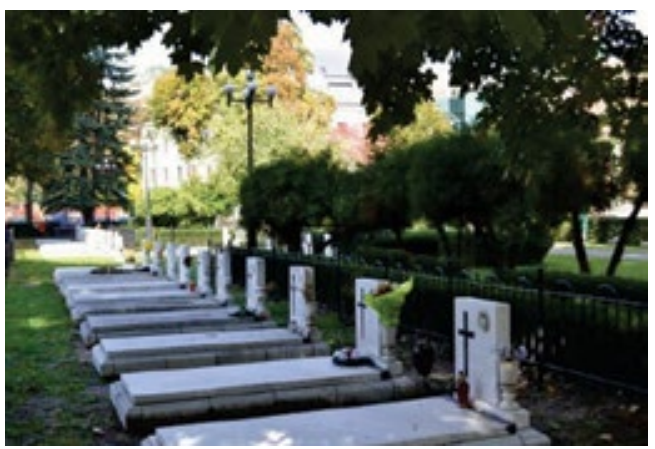

Figure 10. Brasov, centre-ville.

\section{Conclusions}

Dans des registres esthétiques divers, les cinéastes roumains, en réactualisant des événements traumatiques pour leur génération, ont proposé quelques interprétations et surtout ont soulevé des questions, qui restent ouvertes aujourd'hui. Leur mise en question de l'Histoire reflète la quête identitaire douloureuse de cette génération qui cherche encore de se redéfinir et de trouver des repères. En réponse à l'Histoire, avec ses déraillements et ses inconnues, et à un système totalitaire qui avait détruit systématiquement la vie quotidienne des gens, les cinéastes roumains ont présenté leur point de vue et leur vision sur les choses, à travers des histoires mineures, émouvantes, humaines. Au-delà des films discutés ici, le questionnement sur l'«Homme nouveau» se suit, car cette génération extraordinaire des cinéastes roumains continuent leurs interrogations et leur recherche des réponses dans d'autres films, plus récents. Par exemple, en changeant de registre esthétique, Cristian Mungiu continue sa trilogie annoncée, avec un film épisodique et collectif, dont il écrit également le scénario, et qui est une sorte de comédie amère portant sur quelques légendes urbaines durant le communisme, et où les autres réalisateurs sont Ioana Uricaru, Hanno Höfer, Razvan Marculescu et Constantin Popescu. Le titre,
Souvenirs de l'Époque d'or, pourrait devenir générique pour tous les films qu'on a vus ici, car il synthétise l'une des préoccupations primordiales de cette génération: le rapport problématique avec le passé récent et ses traumatismes et le devoir de se souvenir. Je crois que l'une des forces de ce cinéma réside justement dans cette prise de position devant l'Histoire. Dans une analyse politique de cette période de l'histoire roumaine, en réfléchissant sur la question de la mémoire, Vladimir Tismaneanu [3] affirme : «In the process of dealing with the communist past there is no surplus of memory. To forgive doesn't mean to forget, but to forget is simply impardonable.» $\mathrm{Si}$ en Roumanie, depuis les évènements de décembre 1989, tant la société politique que la société civile oscillent entre amnésie et hypermnésie - définie par Alain Besançon [4] comme un "déséquilibre entre la conscience collective d'un fait historique et celle d'autres faits contemporains qui, eux, sont l'objet d'amnésie collective» - avec ces films on voit clairement qu'oublier n'est pas une thérapie et que la génération de l'«Homme nouveau», obligée de se taire durant les années de dictature, fait de l'acte de se souvenir un devoir que les cinéastes assument pleinement.

\section{References}

1) Siegfried Kracauer, Theory of film. The redemption of physical reality, edited by Princeton University Press (Princeton, New Jersey, 1997), pages 305-6.

2) Ruxandra Cesereanu, "Timisoara 15-20 décembre 1989 et Bucarest 21-22 décembre 1989", in Communisme, no. 91/92 (2007), 161-172, page 161 .

3) Vladimir Tismaneanu, "Coming to terms with a traumatic past: reflections on democracy, atonement, and memory", in History of Communism in Europe, Politics of Memory in Post-communist Europe, edited by Zeta books, vol. I. (2010), 15-20, page 18.

4) Alain Besançon, Le malheur du siècle : sur le communisme, le nazisme et l'unicité de la Shoah, edited by Fayard (Paris, 1998), page 165 